\title{
Technical note: Inference in hydrology from entropy balance considerations
}

\author{
Stefan J. Kollet ${ }^{1,2}$ \\ ${ }^{1}$ IBG-3, Institute for Bio- and Geosciences, Research Centre Jülich, Jülich, Germany \\ ${ }^{2}$ Centre for High-Performance Scientific Computing in Terrestrial Systems, Geoverbund ABC/J, Jülich, Germany \\ Correspondence to: Stefan J. Kollet (s.kollet@fz-juelich.de)
}

Received: 26 April 2015 - Published in Hydrol. Earth Syst. Sci. Discuss.: 29 May 2015

Revised: 7 June 2016 - Accepted: 13 June 2016 - Published: 12 July 2016

\begin{abstract}
In this study, the method of inference of macroscale thermodynamic potentials, forces, and exchange coefficients for variably saturated groundwater flow is outlined based on the entropy balance. The theoretical basis of the method of inference is the explicit calculation of the internal entropy production from microscale, thermodynamic flux-force relationships using, e.g., hyper-resolution variably saturated groundwater flow models. Emphasis is placed on the two-scale nature of the entropy balance equation that allows simultaneously incorporating movement equations at the micro- and macroscale. The method is illustrated with simple hydrologic cross sections at steady state and periodic sources/sinks at dynamic equilibrium, and provides a thermodynamic point of view of upscaling in variably saturated groundwater flow. The current limitations in the connection with observable variables and predictive capabilities are discussed, and some perspectives for future research are provided.
\end{abstract}

\section{Introduction}

The current earth science literature indicates that entropy balance considerations have been mainly applied in the context of optimality and self-organization. Theories of optimality and self organization are appealing when dealing with complex nonlinear systems, because of their apparent usefulness in interpreting interactions of gradients and fluxes and in quantifying (predicting) systems' states and uncertainties. In this context, the entropy and energy balance received attention, because of its physics-based foundation in nonequilibrium thermodynamics and potential connection with infor- mation theory (e.g., Dewar, 2003; Koutsoyiannis, 2014). The entropy balance appears to be useful in applications to hydrologic (e.g., Zehe et al., 2013; Ehret et al., 2014), ecohydrologic (e.g., Dewar, 2010; Miedziejko and Kedziora, 2014; del Jesus et al., 2012), and atmospheric sciences (e.g., Paillard and Herbert, 2013), and in general to open complex nonlinear thermodynamic systems (Abe and Okuyama, 2011).

The entropy balance states that in an open system, the change in entropy equals the internal production of entropy minus the divergence of the entropy current. A dynamic equilibrium or steady state is obtained, when entropy production inside (due to, e.g., flow processes of heat or matter) equals the divergence of the entropy current, i.e., the entropy exchange with the outside. Note also, dynamic equilibrium refers to a state of stationarity in the statistical sense. Optimality of the dynamic equilibrium may be achieved, because the gradient, which drives the flux and, thus, the production of entropy, is reciprocally depleted by the same flux (Kleidon et al., 2013).

In hydrology, the entropy balance has been applied to conceptual problems based on the overarching rationale that entropy production is maximized (maximum entropy production, MEP) in obtaining a state of dynamic equilibrium by optimizing the fluxes and gradients in competition via an adjustment of some (non)linear exchange coefficient. There have been some studies demonstrating how entropy production can be maximized by optimizing an exchange coefficient to obtain an optimal system's state. In hydrology, there are quite a few examples of the application and discussion of the MEP principle (e.g., Ehret et al., 2014; Westhoff et al., 2014; Kleidon and Schymanski, 2008) also in connection with data (e.g., Zehe et al., 2013). However, its validity and applicabil- 
ity to hydrologic systems is still in question (Westhoff and Zehe, 2013).

Often the entropy balance has been applied at steady state with simple bucket models, which are well mixed (i.e., without internal gradients). For example, Porada et al. (2011) performed a detailed entropy production analysis of the land surface hydrologic cycle including the shallow vadose zone assuming vertical equilibrium of the soil bucket model. Applying linear bucket models without considering internal gradients, Kleidon and Schymanski (2008) showed that if the natural system possesses enough degrees of freedom, in case of steady state, the system will tend towards a certain exchange coefficient, when entropy production is maximized. For similar bucket models, Westhoff et al. (2014) demonstrated the impact of periodic boundary forcing on entropy production, which may result in more than one maximum for unique values of the exchange coefficient at dynamic equilibrium. Interestingly, these studies did not calculate the internal entropy production explicitly. Instead, entropy production was estimated indirectly from the exchange with the outside (i.e., the divergence of the entropy current).

In order to optimize effective values of a simple two-box model, Schymanski et al. (2010) recognized the potential of explicitly estimating the internal entropy production using a simple distributed model of the water and carbon balance (Klausmeier, 1999), which is based on coupled equations of moisture and biomass and is able to produce vegetation patterns. This study highlights an interesting aspect of entropy balance considerations that is the inference of upscaled effective parameters and state variables to represent subgrid scale variability in coarse scale (macroscale) models. Thus, ultimately, the appeal of the entropy balance maybe the inference of upscaled or effective exchange coefficients and forces/gradients, which may be used to quantitatively describe the complex system without the explicit knowledge about microscopic details (Dewar, 2009). In this context, a popular example is gas diffusion, which can be captured by an inferred, macroscopic diffusion coefficient and gradient instead of honoring the motion and interactions of individual molecules.

In this study, the method of inference of effective hydrologic exchange coefficients, potentials and forces is outlined using the entropy balance equation in applications to simple hydrologic cross sections. The purpose of this study is to direct attention to the potential insights gained from a new branch of theoretical hydrology combining modern thermodynamic principles with numerical experiments. While the thermodynamic principles constitute the link between different spatial scales that may be useful in upscaling hydrologic process across a hierarchy of scales, the numerical experiments constitute the methodological pillar to obtain explicitly the internal entropy production or dissipation required in the upscaling, equivalent to ab initio simulations in molecular dynamics (Kresse and Hafner, 1994). The following sections provide the basic theory with an emphasis on the two-scale nature of the entropy balance, and the application to the hydrologic cross sections with ensuing discussion and conclusions.

\section{Basic theory and the two-scale nature of the entropy balance}

The theory outlined in Kondepudi and Prigogine (2014) is applied to the problem of variably saturated groundwater flow at constant temperature. Based on conservation of energy (and the balance equation for concentrations, which is not required in this analysis) Kondepudi and Prigogine (2014) write the entropy balance as follows:

$s^{\prime}+\nabla \cdot J_{\mathrm{s}}=\sigma$,

where $s^{\prime}\left(\mathrm{ML}^{-1} \mathrm{~T}^{-3} \mathrm{~K}^{-1}\right)$ is the change in the entropy density with time; $J_{\mathrm{S}}\left(\mathrm{MT}^{-3} \mathrm{~K}^{-1}\right)$ is the entropy current per unit of volume; and $\sigma\left(\mathrm{ML}^{-1} \mathrm{~T}^{-3} \mathrm{~K}^{-1}\right)$ is the internal entropy production per unit of volume, which is always positive by definition. Thus, the change of entropy density with time of a macroscopic volume depends on the divergence of the entropy current and the internal entropy production.

In the considered case of variably saturated groundwater flow, $J_{\mathrm{s}}=J M / T$, where $J\left(\mathrm{ML}^{-2} \mathrm{~T}^{-1}\right)$ is the mass flow per unit area, $M\left(\mathrm{~L}^{2} \mathrm{~T}^{-2}\right)$ is the chemical potential (i.e., the sum of pressure and gravitational potential, Eq. 5) at the macroscale and $T(\mathrm{~K})$ is the temperature. At the microscale, defining $q\left(\mathrm{ML}^{-3} \mathrm{~T}^{-1}\right)$ and $f\left(\mathrm{~L}^{2} \mathrm{~T}^{-2}\right)$ as the fluxes and thermodynamic forces per unit of volume, the divergence of the entropy current and the internal entropy production can be expanded as follows:

$s^{\prime}+(M / T)(\nabla \cdot J)+J \cdot(\nabla(M / T))=\sum q f / T$.

For the derivations below it is important to recognize that Eq. (2) exhibits the unique characteristics of incorporating two scales: the entropy density change with time and divergence of the entropy current at the macroscale (all terms on the left-hand side), and the entropy production at the microscale, i.e., the sum of all products of the internal microscopic fluxes and forces (term on the right-hand side). Note, in the following, the temperature $T(\mathrm{~K})$ is omitted in the equations and units, because $T$ is constant in the following derivations.

Performing an entropy balance at steady state leads to

$M(\nabla \cdot J)+J \cdot(\nabla M)=\sigma$

because $s^{\prime}=0$. In contrast, performing an entropy balance under the influence of periodic external forcing requires integration over one full forcing cycle at dynamic equilibrium of Eq. (2) indicated by overbars

$\overline{M(\nabla \cdot J)}+\overline{J \cdot(\nabla M)}=\bar{\sigma}$ 
with $\overline{s^{\prime}}=0$ over one full cycle. Both approaches will be applied in the following sections, in order to arrive at effective variables at the macroscale.

Because of the two-scale nature of Eqs. (1) and (2), movement equations are introduced at the macroscale and microscale. At the macroscale, $M\left(\mathrm{~L}^{2} \mathrm{~T}^{-2}\right)$ is defined as the sum of the macroscopic pressure potential $\Psi\left(\mathrm{L}^{2} \mathrm{~T}^{-2}\right)$, and gravitational potential $g z\left(\mathrm{~L}^{2} \mathrm{~T}^{-2}\right)$, leading to

$M=\Psi+g z$,

and is, thus, equivalent to the hydraulic head; $(\nabla M)$ symbolizes a macroscopic thermodynamic force $F\left(\mathrm{~L}^{2} \mathrm{~T}^{-2}\right)$ as the difference in the macroscopic chemical potentials

$(\nabla M)=F=M_{\text {high }}-M_{\text {low }}$

and, at the moment, $J$ is defined as a conductance concept

$J=\lambda F$,

where $\lambda\left(\mathrm{ML}^{-4} \mathrm{~T}\right)$ is a conductance coefficient $\left(\lambda=\rho r_{\mathrm{s}}\right.$, with water density $\rho\left(\mathrm{ML}^{-3}\right)$ and resistance $\left.r_{\mathrm{s}}\left(\mathrm{TL}^{-1}\right)\right)$ relating the flux with the force at the macroscale.

At the microscale, the chemical potential $\mu,\left(\mathrm{L}^{2} \mathrm{~T}^{-2}\right)$, the mass flux $q\left(\mathrm{ML}^{-3} \mathrm{~T}^{-1}\right)$ per unit of volume and the thermodynamic force $f\left(\mathrm{~L}^{2} \mathrm{~T}^{-2}\right)$ are

$\mu=\psi+g z$

where $\psi\left(\mathrm{L}^{2} \mathrm{~T}^{-2}\right)$ is the microscale pressure potential;

$q=\frac{1}{\alpha} \rho \frac{K}{v} k_{\mathrm{r}}(\psi)\left(\mu_{\mathrm{high}}-\mu_{\mathrm{low}}\right)$,

where $\rho\left(\mathrm{ML}^{-3}\right)$ is the density; $v\left(\mathrm{~L}^{2} \mathrm{~T}^{-1}\right)$ is the kinematic viscosity; $K$ is the permeability $\left(\mathrm{L}^{2}\right), k_{\mathrm{r}}(\psi)(-)$ is the relative permeability, and $\alpha\left(\mathrm{L}^{-2}\right)$ is the unit microscopic flowthrough area; and the microscale force is

$f=\left(\mu_{\text {high }}-\mu_{\text {low }}\right)$.

Technically, $\sum q f$ is the sum of all fluxes and forces (always positive because any flux produces entropy) between all neighboring cells or elements in a microscale, numerical, variably saturated groundwater flow model including Dirichlet and/or Neumann boundary conditions.

Thus, the two-scale nature of Eq. (2) allows to apply different thermodynamic flux-force relationships at the different scales that are the conductance concept at the macroscale (Eq. 7) and essentially Darcy's law or Richards' equation (Eq. 9) at the microscale. In Eq. (2), the entropy production serves as an automatic spatial and also temporal integrator of the microscale fluctuations. These two characteristics are remarkable. Note, the calculation (integration) of the entropy balance may be performed over the global domain of volume $V\left(\mathrm{~L}^{3}\right)$ or any subdomain $V_{i}\left(\mathrm{~L}^{3}\right)$ thereof.

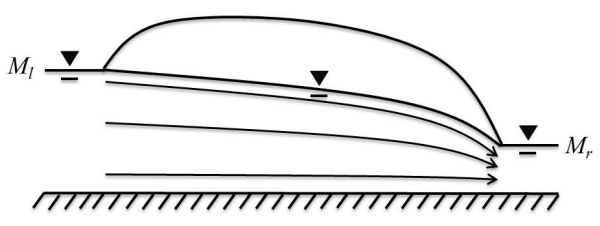

Figure 1. Schematic of a simple profile with Dirichlet boundary conditions on the right and left $\left(M_{\mathrm{r}}, M_{1}\right)$ and steady-state, variably saturated flow. In the theory, the vertical and horizontal extents of the cross section are assumed to be constant.

\section{Method of inference}

The basis of the method of inference is that the internal, microscopic entropy production $\sigma$ and also the complete entropy balance can be calculated from support scale simulations by implementing the microscale Eqs. (9) and (10) in combination with a continuity equation over the macroscopic domain. In obtaining $\sigma$ explicitly, one is able to estimate effective potentials, forces, and conductance coefficients of Eq. (7) at the macroscale from the explicitly resolved fluctuations at the microscale, which are thermodynamically consistent. In order to illustrate the method of inference of macroscale potentials, conductances, and forces, a number of illustrative examples based on simple hydrologic profiles are presented applying different boundary conditions and source/sink terms.

\subsection{Example 1}

Directed at a heat flow example in Kondepudi and Prigogine (2014), a simple cross section is considered (Fig. 1) with steady-state, variably saturated groundwater flow, $J$, from left to right due to Dirichlet boundary conditions on the left $M_{\mathrm{r}}$, and right $M_{\mathrm{l}}$, with $M_{\mathrm{l}}>M_{\mathrm{r}}$. Because $\nabla \cdot J=0$, and $s^{\prime}=0$ at steady state, integration of the entropy balance over the cross section leads to

$$
\begin{aligned}
& S_{i}=\int_{0}^{L_{z}} \int_{0}^{L_{x}} \sigma(x, z) d x d z=L_{z} \int_{0}^{L_{x}} J_{x}\left(\nabla_{x} M\right) d x \\
& S_{i}=L_{z} J_{x}\left(M_{1}-M_{\mathrm{r}}\right)=L_{z} J_{x} F,
\end{aligned}
$$

where $L_{z}$ and $L_{x}(L)$ are the constant vertical and horizontal extents of the cross section, respectively; $S_{i}$ is the total internal entropy production; and $F=\left(M_{1}-M_{\mathrm{r}}\right)$ is the macroscopic force. Note, in the following, the entropy production integral is simply written as $S_{i}=\int \sigma$, and $L_{z}$ is lumped into the flux $L_{z} J_{x}=J$ for convenience.

In case of this simple example, applying $J=\lambda\left(M_{1}-M_{\mathrm{r}}\right)$ from Eq. (5), one obtains the expression for the effective conductance

$\lambda=S_{i}\left(M_{1}-M_{\mathrm{r}}\right)^{-2}=S_{i} F^{-2}$ 


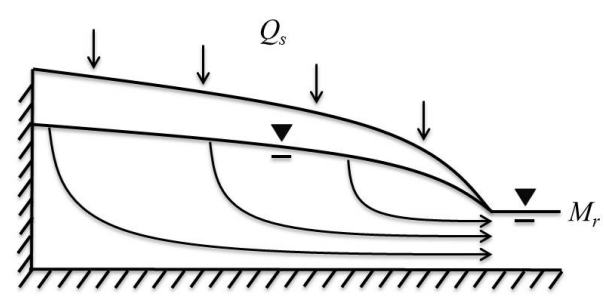

Figure 2. Schematic of a simple profile with a Dirichlet boundary condition on the right $\left(M_{\mathrm{r}}\right)$, a no-flow boundary condition on the left, a constant source $\left(Q_{\mathrm{s}}\right)$, and steady-state, variably saturated groundwater flow. In the theory, the vertical and horizontal extents of the cross section are assumed to be constant.

and the effective force

$F=S_{i} J^{-1}$.

Thus, one may obtain the effective conductance for any kind of heterogeneity (i.e., microscale fluctuations) by explicitly calculating $\sigma$ and $S_{i}$ based on Eqs. (6) and (7) and the macroscopic boundary conditions $M_{1}$ and $M_{\mathrm{r}}$. Note, entropy production is simply the sum of the product of the steady-state fluxes and incremental forces over the cross section $S_{i}=\int \sigma=\int\left(\sum q f\right)=$ $\int\left(\sum \frac{1}{\alpha} \rho \frac{K}{v} k_{\mathrm{r}}(\psi)\left(\mu_{\text {high }}-\mu_{\text {low }}\right)^{2}\right)$, where individual values of $q f$ are calculated with Eqs. (9) and (10) between two adjacent microscale elements in support scale numerical simulations. While $\lambda$ could have been obtained directly from the macroscopic flux and the applied boundary conditions similar to a numerical Darcy experiment, the example serves to illustrate the basic concept of inference arriving at a thermodynamic expression for $\lambda$ and the force $F$ (if a flux is prescribed at the boundaries).

\subsection{Example 2}

This example expands example 1 to steady-state groundwater flow including recharge represented by the mass rate $Q_{\text {s }}$

$$
Q_{\mathrm{s}}=\int_{0}^{L}(\nabla \cdot J) d x,
$$

and integration leading to

$M Q_{\mathrm{s}}+J_{1} M_{1}-J_{\mathrm{r}} M_{\mathrm{r}}=S_{i}$,

where $M$ is the macroscopic potential of the cross section.

The general expression for the macroscopic potential of the cross section is

$M=Q_{\mathrm{s}}^{-1}\left(S_{i}-\left(J_{1} M_{1}-J_{\mathrm{r}} M_{\mathrm{r}}\right)\right)$.

In this example, three special cases are considered, namely $J_{1}=0, J_{1}<0$, and $J_{1}>0$. In case of $J_{1}=0$ (Fig. 2), there

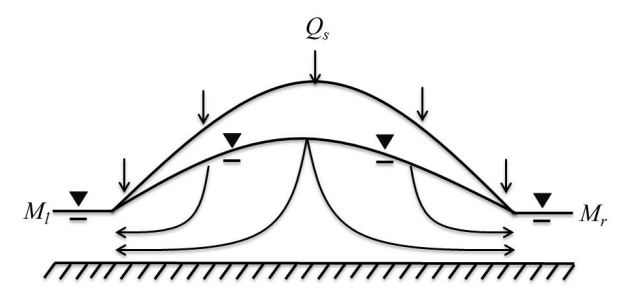

Figure 3. Schematic of a simple profile with Dirichlet boundary conditions on the right and left $\left(M_{\mathrm{r}}, M_{\mathrm{l}}\right)$ a constant source $\left(Q_{\mathrm{S}}\right)$, and steady-state, variably saturated groundwater flow. In this symmetric case, there exists a water divide in the center of the domain. In the theory, the vertical and horizontal extents of the cross section are assumed to be constant.

is a no-flow boundary condition on the left side resulting in $J_{\mathrm{r}}=Q_{\mathrm{s}}$ and, thus

$M=S_{i, J_{1}=0} Q_{\mathrm{s}}^{-1}+M_{\mathrm{r}}$

$F=\left(M-M_{\mathrm{r}}\right)=S_{i, J_{1}=0} Q_{\mathrm{s}}^{-1}$,

where the subscript indicates the respective case for the left boundary flux.

With Eq. (7) and $J_{\mathrm{r}}=Q_{\mathrm{s}}=J$,

$\lambda=S_{i, J_{1}=0} F^{-2}$

follows for the conductance coefficient.

For $J_{1}<0$ (Fig. 3), the symmetric case is considered, where the potentials at the boundaries are equal $\left(M_{\mathrm{l}}=M_{\mathrm{r}}=M_{\mathrm{b}}\right)$ and $Q_{\mathrm{s}}$ is uniform over the profile $\left(-J_{1}=J_{\mathrm{r}}=Q_{\mathrm{s}} / 2\right)$ leading to

$M Q_{\mathrm{s}}-1 / 2 Q_{\mathrm{s}} M_{1}-1 / 2 Q_{\mathrm{s}} M_{\mathrm{r}}=S_{i, J_{\mathrm{l}}<0}$

$Q_{\mathrm{s}}\left(M-\left(M_{1}+M_{\mathrm{r}}\right) / 2\right)=S_{i, J_{1}<0}$

$Q_{\mathrm{s}}\left(M-M_{\mathrm{b}}\right)=S_{i, J_{1}<0}$,

and ultimately for the macroscopic potential

$M=S_{i, J_{1}<0} Q_{\mathrm{s}}^{-1}+M_{\mathrm{b}}$.

$F=\left(M-M_{\mathrm{b}}\right)=S_{i, J_{1}<0} Q_{\mathrm{s}}^{-1}$,

and

$\lambda=S_{i, J_{1}<0} F^{-2}$.

Note, $M$ and $F$ reflect values for each of the two half spaces separated by a no-flow boundary condition, e.g., $F=$ $\left(S_{i, J_{1}<0} / 2\right)\left(Q_{\mathrm{s}} / 2\right)^{-1}$, which is true for a homogeneous profile only and is equivalent to the case $J_{1}<0$ above. The entropy production is calculated also with $S_{i, J_{1}<0}=\int \sigma=$ $\int\left(\sum q f\right)=\int\left(\sum \frac{1}{\alpha} \rho \frac{K}{v} k_{\mathrm{r}}(\psi)\left(\mu_{\text {high }}-\mu_{\text {low }}\right)^{2}\right)$.

For a heterogeneous profile and/or $M_{1}>M_{\mathrm{r}}$ (Fig. 4), i.e., when there is no symmetry

$M Q_{\mathrm{s}}-J_{1} M_{1}-J_{\mathrm{r}} M_{\mathrm{r}}=S_{i, J_{1}<0}$. 


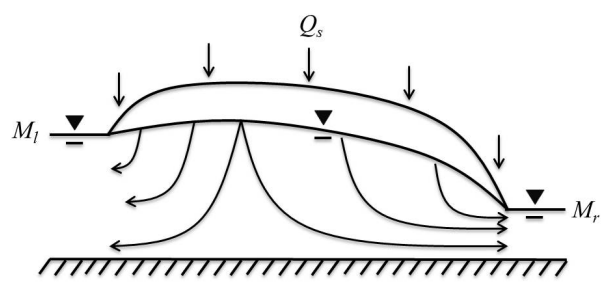

Figure 4. Schematic of a simple profile with Dirichlet boundary conditions on the right and left $\left(M_{\mathrm{r}}, M_{1}\right)$ a constant source $\left(Q_{\mathrm{s}}\right)$, and steady-state, variably saturated flow. In this case, there exists a water divide in the domain. In the theory, the vertical and horizontal extent of the cross section is assumed to be constant.

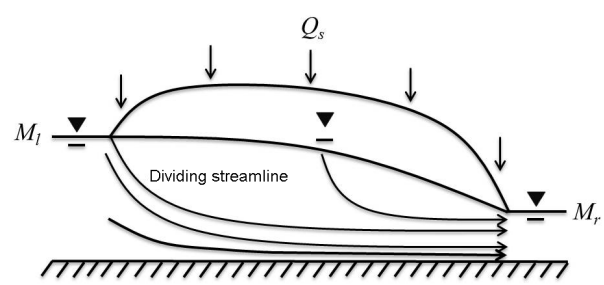

Figure 5. Schematic of a simple profile with Dirichlet boundary conditions on the right and left $\left(M_{\mathrm{r}}, M_{1}\right)$, a constant source $\left(Q_{\mathrm{S}}\right)$, and steady-state, variably saturated groundwater flow. Note the dividing streamline in this example. In the theory, the vertical and horizontal extents of the cross section are assumed to be constant.

Thus, the effective potential $M$ of the cross section may be obtained from

$M=Q_{\mathrm{s}}^{-1}\left(S_{i, J_{1}<0}+J_{1} M_{1}+J_{\mathrm{r}} M_{\mathrm{r}}\right)$.

Additionally, expressions can be obtained for the conductance coefficients in the exchange with the left and right boundary conditions that are

$\lambda_{1}=\left(M Q_{\mathrm{s}}-S_{i, J_{1}<0}-J_{\mathrm{r}} M_{\mathrm{r}}\right)\left(F_{1} M_{\mathrm{l}}\right)^{-1}$

$\lambda_{\mathrm{r}}=\left(M Q_{\mathrm{s}}-S_{i, J_{1}<0}-J_{1} M_{\mathrm{l}}\right)\left(F_{\mathrm{r}} M_{\mathrm{r}}\right)^{-1}$

where the macroscale forces $F_{\mathrm{r}}=M-M_{\mathrm{r}}$ and $F_{1}=M-$ $M_{1}$ result from the differences between $M$ and $M_{1}$, $M_{\mathrm{r}}$ with $M$ following from Eq. (25). Again, entropy production is calculated with $S_{i, J_{1}<0}=\int \sigma=\int\left(\sum q f\right)=$ $\int\left(\sum \frac{1}{\alpha} \rho \frac{K}{v} k_{\mathrm{r}}(\psi)\left(\mu_{\text {high }}-\mu_{\text {low }}\right)^{2}\right)$.

For $J_{1}>0$ (Fig. 5), the entropy balance is

$M Q_{\mathrm{s}}+J_{1} M_{1}-J_{\mathrm{r}} M_{\mathrm{r}}=S_{i, J_{1}>0}$

and the macroscopic potential is

$M=Q_{\mathrm{s}}^{-1}\left(S_{i, J_{1}>0}-J_{1} M_{1}+J_{\mathrm{r}} M_{\mathrm{r}}\right)$.

With $Q_{\mathrm{s}}=J_{\mathrm{r}}-J_{1}$, follows

$J_{1}\left(M_{1}-M\right)+J_{\mathrm{r}}\left(M-M_{\mathrm{r}}\right)=S_{i, J_{1}>0}$.

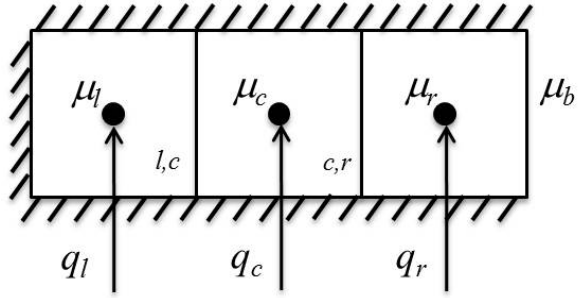

Figure 6. Schematic of the discrete example consisting of three microscale elements with a Dirichlet boundary condition on the right side $\left(\mu_{\mathrm{b}}\right)$ and a source/sink in each element $\left(q_{1}, q_{\mathrm{c}}, q_{\mathrm{r}}\right)$.

Thus, two conductances can be obtained, which are

$\lambda_{1}=\left(S_{i, J_{\mathrm{l}}>0}-J_{\mathrm{r}}\left(M-M_{\mathrm{r}}\right)\right) F_{1}^{-2}$

$\lambda_{\mathrm{r}}=\left(S_{i, J_{1}>0}-J_{1}\left(M_{1}-M\right)\right) F_{\mathrm{r}}^{-2}$

with the macroscopic forces $F_{1}=\left(M_{1}-M\right)$ and $F_{\mathrm{r}}=$ $\left(M-M_{\mathrm{r}}\right)$. In this example, two additional conductances can be obtained for the subdomains separated by the dividing streamline due to recharge shown in Fig. 5 that are

$\lambda_{Q_{\mathrm{s}}}=\left(S_{i, J_{1}>0}-J_{1}\left(M_{1}-M_{\mathrm{r}}\right)\right) F_{Q_{\mathrm{s}}}^{-2}$

$\lambda_{l, r}=\left(S_{i, J_{1}>0}-Q_{\mathrm{s}}\left(M-M_{\mathrm{r}}\right)\right) F_{l, r}^{-2}$,

with $J_{\mathrm{r}}=J_{1}+Q_{\mathrm{s}}$, and the macroscale forces $F_{Q_{\mathrm{s}}}=$ $\left(M-M_{\mathrm{r}}\right)$ and $F_{1, \mathrm{r}}=\left(M_{1}-M_{\mathrm{r}}\right)$. In the domain, the entropy production is calculated also with $S_{i, J_{1}>0}=\int \sigma=$ $\int\left(\sum q f\right)=\int\left(\sum \frac{1}{\alpha} \rho \frac{K}{v} k_{\mathrm{r}}(\psi)\left(\mu_{\text {high }}-\mu_{\text {low }}\right)^{2}\right)$.

\subsection{Example 3}

In this example, a no-flow boundary condition on the left is considered resembling a hillslope with a no-flow boundary along a hypothetical ridge on the left side, and a Dirichlet boundary condition along a hypothetical stream on the right side. Now, a source/sink $Q_{\mathrm{s}}(x, t)$ varies periodically in space and time (periodically varying recharge/discharge). In this case, Eq. (2) needs to be solved for the different variables and integrated over one complete cycle at dynamic equilibrium.

Note, again $\int_{0}^{L} \nabla \cdot J d x=Q_{\mathrm{s}}$ because there is a macroscopic, transient source/sink in the domain, therefore, after integration along the cross section, the entropy balance reads

$S^{\prime}+M Q_{\mathrm{s}}-J_{\mathrm{r}} M_{\mathrm{r}}=S_{i}$,

where $S^{\prime}$ is the entropy change rate. After time integration over one full cycle at dynamic equilibrium, $\overline{Q_{\mathrm{s}}}=0$ and $\overline{S^{\prime}}=$ 0 , the effective macroscopic potential of the cross section due to the periodic varying source/sink is

$\bar{M}=\overline{\left(S_{i}+J_{\mathrm{r}} M_{\mathrm{r}}-S^{\prime}\right) Q_{\mathrm{s}}^{-1}}$ 
or

$\bar{M}=\operatorname{cov}\left(S_{i}, Q_{\mathrm{s}}^{-1}\right)+\overline{S_{i}} \overline{Q_{\mathrm{s}}^{-1}}+$

$M_{\mathrm{r}}\left(\operatorname{cov}\left(J_{\mathrm{r}}, Q_{\mathrm{s}}^{-1}\right)+\overline{J_{\mathrm{r}}} \overline{Q_{\mathrm{s}}^{-1}}\right)+\operatorname{cov}\left(S^{\prime}, Q_{\mathrm{s}}^{-1}\right)$,

based on the definition of the covariance.

Recognizing that $J_{\mathrm{r}}=\int_{0}^{L}\left(Q_{\mathrm{s}}-\Theta^{\prime}\right) d x$, where $\Theta^{\prime}$ is the macroscopic mass change rate of the cross section, one obtains for the effective force

$$
\bar{F}=\overline{\left(S_{i}-\Theta^{\prime} M_{\mathrm{r}}-S^{\prime}\right) Q_{\mathrm{s}}^{-1}}
$$

or

$$
\begin{aligned}
& \bar{F}=\operatorname{cov}\left(\sigma, Q_{\mathrm{s}}^{-1}\right)+\bar{\sigma} \overline{Q_{\mathrm{s}}^{-1}}-M_{\mathrm{r}} \operatorname{cov}\left(\Theta^{\prime}, Q_{\mathrm{s}}^{-1}\right) \\
& -\operatorname{cov}\left(S^{\prime}, Q_{\mathrm{s}}^{-1}\right),
\end{aligned}
$$

with $\overline{\Theta^{\prime}}=0$ due to dynamic equilibrium; and for the effective conductance

$\bar{\lambda}=\overline{\left(S_{i}-\Theta^{\prime} M-S^{\prime}\right) F^{2}}$

or

$\bar{\lambda}=\operatorname{cov}\left(S_{i}, F^{2}\right)+\overline{S_{i}} \overline{F^{2}}-\operatorname{cov}\left(\Theta^{\prime} M, F^{2}\right)$

$+\overline{\Theta^{\prime} M} \overline{F^{2}}+\operatorname{cov}\left(S^{\prime}, F^{2}\right)$

with $J_{\mathrm{r}}=\lambda F=\lambda\left(M-M_{\mathrm{r}}\right)$.

Apparently, on the right-hand side of Eqs. (35), (36), and (37) all terms may be calculated from the numerical simulations except the entropy change rate $S^{\prime}=\int s^{\prime}$ and therefore also $\operatorname{cov}\left(S^{\prime}, Q_{\mathrm{s}}^{-1}\right)$, because both, $S^{\prime}$ and $M$ are not known in Eq. (34) (note, $S_{i}$ is calculated explicitly). However, $S^{\prime}$ is needed in the estimation of $\bar{F}$ and $\bar{\lambda}$ and may actually be calculated from the microscale variables, which is demonstrated with a discrete example depicted in the schematic in Fig. 6.

In this schematic, there are three microscale elements with sources/sinks in each individual element $\left(q_{1}, q_{\mathrm{c}}, q_{\mathrm{r}}\right)$ and a constant potential boundary condition on the right $\left(\mu_{\mathrm{b}}\right)$. For each individual element the entropy balance is

$$
\begin{aligned}
& s_{1}^{\prime}+q_{1} \mu_{1}-q_{1, \mathrm{c}} \mu_{1, \mathrm{c}}=\sum q_{1} f_{1}=q_{1, \mathrm{c}}\left(\mu_{1}-\mu_{1, \mathrm{c}}\right) \\
& s_{\mathrm{c}}^{\prime}+q_{\mathrm{c}} \mu_{\mathrm{c}}+q_{1, \mathrm{c}} \mu_{1, \mathrm{c}}-q_{\mathrm{c}, \mathrm{r}} \mu_{\mathrm{c}, \mathrm{r}}=\sum q_{\mathrm{c}} f_{f} \\
& =q_{1, \mathrm{c}}\left(\mu_{1, \mathrm{c}}-\mu_{\mathrm{c}}\right)+q_{\mathrm{c}, \mathrm{r}}\left(\mu_{\mathrm{c}}-\mu_{\mathrm{c}, \mathrm{r}}\right) \\
& s_{\mathrm{r}}^{\prime}+q_{\mathrm{r}} \mu_{\mathrm{r}}+q_{\mathrm{c}, \mathrm{r}} \mu_{\mathrm{c}, \mathrm{r}}-q_{\mathrm{b}} \mu_{\mathrm{b}}=\sum q_{\mathrm{r}} f_{\mathrm{r}}=q_{\mathrm{c}, \mathrm{r}}\left(\mu_{\mathrm{c}, \mathrm{r}}-\mu_{\mathrm{r}}\right) \\
& +q_{\mathrm{b}}\left(\mu_{\mathrm{r}}-\mu_{\mathrm{b}}\right)
\end{aligned}
$$

where the fluxes and potentials with the subscript $1, \mathrm{c}$ and $\mathrm{c}, \mathrm{r}$ are valid at the element interfaces. The terms on the right-hand side, i.e., the entropy production for each element, encompass the fluctuations in the flux-force relationships between the element's interior and the element boundaries.
Summation of the individual balance equations leads to the total balance

$$
\begin{aligned}
& s^{\prime}+q_{1} \mu_{1}+q_{\mathrm{c}} \mu_{\mathrm{c}}+q_{\mathrm{r}} \mu_{\mathrm{r}}-q_{\mathrm{b}} \mu_{\mathrm{b}}=\sigma=q_{1, \mathrm{c}}\left(\mu_{1}-\mu_{\mathrm{c}}\right) \\
& +q_{\mathrm{c}, \mathrm{r}}\left(\mu_{\mathrm{c}}-\mu_{\mathrm{r}}\right)+q_{\mathrm{b}}\left(\mu_{\mathrm{r}}-\mu_{\mathrm{b}}\right) .
\end{aligned}
$$

Note, on the left-hand side, all the interface terms disappear and only the source and boundary terms remain, equivalent to the macroscale balance in Eq. (34). Equation (38) is the entropy balance equation for the system depicted in Fig. 6 .

Any changes in the entropy of the system with time are due to transient effects that cancel out at dynamic equilibrium $\overline{s^{\prime}}=0$. In order to demonstrate this, substitution of $q_{1, \mathrm{c}}=$ $\left(q_{1}-\theta_{\mathrm{l}}{ }^{\prime}\right), q_{\mathrm{c}, \mathrm{r}}=\left(q_{1}-\theta_{\mathrm{l}}{ }^{\prime}\right)+\left(q_{\mathrm{c}}-\theta_{\mathrm{c}}{ }^{\prime}\right)$, and $q_{\mathrm{b}}=\left(q_{1}-\theta_{\mathrm{l}}{ }^{\prime}\right)+$ $\left(q_{\mathrm{c}}-\theta_{\mathrm{c}}{ }^{\prime}\right)+\left(q_{\mathrm{r}}-\theta_{\mathrm{r}}{ }^{\prime}\right)$ for the interface fluxes on the righthand side in Eq. (38) leads to

$$
\begin{aligned}
& s^{\prime}+q_{1} \mu_{1}+q_{\mathrm{c}} \mu_{\mathrm{c}}+q_{\mathrm{r}} \mu_{\mathrm{r}}-q_{\mathrm{b}} \mu_{\mathrm{b}}=\left(q_{1}-\theta_{\mathrm{l}}^{\prime}\right) \mu_{1} \\
& +\left(q_{\mathrm{c}}-\theta_{\mathrm{c}}{ }^{\prime}\right) \mu_{\mathrm{c}}+\left(q_{\mathrm{r}}-\theta_{\mathrm{r}}^{\prime}\right) \mu_{\mathrm{r}}-q_{\mathrm{b}} \mu_{\mathrm{b}},
\end{aligned}
$$

which demonstrates continuity in case of steady-state $\theta_{\mathrm{l}}^{\prime}=$ $\theta_{\mathrm{c}}{ }^{\prime}=\theta_{\mathrm{r}}{ }^{\prime}=0$, and shows that any, e.g., positive mass storage change $\theta^{\prime}$ over the microscopic volume leads to negative change in entropy and vice versa. Note, the entropy production is still always positive as required by definition. Thus, $S^{\prime}$ and $M$ can be evaluated by applying Eq. (39) to microscale simulations.

A special case may be considered, in which the system depicted in Fig. 6 is also closed on the right side resulting in a sole exchange with the surroundings via the periodic source/sink (e.g., infiltration/evapotranspiration) $Q_{\mathrm{s}}(t)$. This would be equivalent to a profile with a discharge area in the center and the assumption of symmetry shown in the schematic in Fig. 7. The requirement again is that $\overline{Q_{\mathrm{s}}}=$ 0 over one full cycle at dynamic equilibrium. Then, e.g., Eq. (35) simplifies to

$$
\bar{M}=\overline{\left(S_{i}-S^{\prime}\right) Q_{\mathrm{s}}^{-1}}
$$

or

$\bar{M}=\operatorname{cov}\left(\Delta S^{\prime}, Q_{\mathrm{s}}^{-1}\right)+\overline{S_{i}} \overline{Q_{\mathrm{s}}^{-1}}$

with $\Delta S^{\prime}=S_{i}-S^{\prime}$.

\section{Discussion}

The major advantage of the proposed inference theory is the estimation of macroscopic variables that are thermodynamically consistent with the microscale fluctuations. This is discussed in the context of the simple example 1 interpreting the entropy current $J_{\mathrm{s}}=J M$ as an advective potential flux. Because $J$ is constant and $M_{1}>M_{\mathrm{r}}$, the entropy current leaving the domain on the right side is smaller than the entropy current entering the domain on the left side. This is 


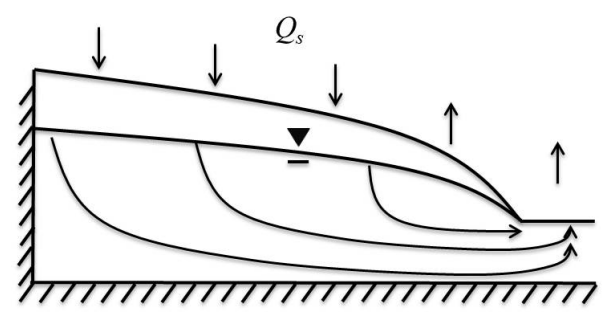

Figure 7. Schematic of a simple profile with a no-flow boundary condition on the left and right (based on symmetry) and transient, spatially varying sources/sinks $Q_{\mathrm{s}}(x, t)$ resulting in a recharge and discharge area. In the theory, the vertical and horizontal extent of the cross section is assumed to be constant.

due to dissipation in the interior of the domain resulting in the production of entropy $S_{i}$. In hydrology, the dissipation is simulated using Darcy's law and Richards' equation at the support (here microscopic) scale, where all dissipative processes are lumped in the hydraulic conductivity representing the flow resistance. Thus, at the macroscale the derived conductance $\lambda$ is thermodynamically consistent if one accepts, e.g., Darcy's law as a valid parameterization of the internal dissipative processes. Note, in this study, $J_{\mathrm{S}}$ is equivalent to the internal energy current in the energy balance equation, because temperature is constant.

Equations (12) and (13) have not been applied before in the context of hydrology. While the equations illustrate the basic idea for the simplest case of a Darcy experiment, one may argue that the insight gained from this example is rather limited, because $\lambda$ could have been obtained from the known flux-force relationship and the conductance equation (one unknown $\lambda$ with one Eq. 7). Examples 2 and 3, on the other hand, clearly illustrate the advantage, because the macroscale potential $M$ (and therefore $F$ ), which are needed to obtain $\lambda$, are not known in these examples. Thus, one is left with two unknowns, $\lambda$ and $F$, and only one equation (the conductance Eq. 7). In the proposed theory, the entropy balance provides the second equation to solve for the two unknowns at the cost of explicitly calculating the internal entropy production $S_{i}=\int \sigma=$ $\int\left(\sum q f\right)=\int\left(\sum \frac{1}{\alpha} \rho \frac{K}{v} k_{\mathrm{r}}(\psi)\left(\mu_{\text {high }}-\mu_{\text {low }}\right)^{2}\right)$, and at the benefit of thermodynamic consistency. This is the central message of the proposed method of inference, which exploits the internal entropy production $S_{i}$ as a spatial and also temporal integrator.

It is important to emphasize that one can also obtain, in an ad hoc fashion, the forces and conductance coefficients for any subdomain $V_{i}$ of the global domain with volume $V$. For example, in order to obtain the macroscale potential in the center of the profile of example 1 , one arrives at

$M_{\mathrm{c}}=M_{1}-J^{-1} \int_{V_{i}} \sigma d V_{i}$.
Thus, from $\int_{V_{i}} \sigma d V_{i}$ estimates, one is able to obtain macroscale variables over a hierarchy of scales for different hydrologic configurations similar to the simple examples provided above.

Under purely saturated groundwater flow conditions, the estimates of macroscale variables can be used directly for predictions, because $\lambda$ is constant for the same flow geometries, which is trivial, but important to realize. In case of variably saturated flow and transient conditions (when the flow geometry changes), $\lambda$ is of course not constant and $S_{i}$ will depend in an unknown, nonlinear fashion on the flux $J$ and its variability (example 3 ), which apparently limits the usefulness of the proposed approach. However, universal relationships of $S_{i}(J)$ and $\operatorname{cov}\left(S_{i}, J^{-1}\right)$ can perhaps be obtained from a series of numerical experiments under characteristic hydrologic configurations.

This also brings up the question of whether one is able to establish a connection of the proposed theory with observations of real-world systems. Obviously, $S_{i}$ can not be measured directly in the field utilizing independent experiments, which could, in turn, be used to derive macroscopic thermodynamic forces from flux observations that are more readily available. Thus, utilizing the entropy balance for estimating macroscopic field variables and ensuing predictions appears limited at this point. Yet, this study suggests exploring relationships of measurable field variables and $S_{i}$ utilizing numerical experiments in the future. In turn, under certain conditions, estimates of $S_{i}$ from measurable quantities may be possible. With the help of the extended example 1, this is discussed below.

Assuming a time-varying force, i.e., Dirichlet boundary conditions, temporal integration of Eq. (11) over one full cycle at dynamic equilibrium yields

$\overline{S_{i}}=\overline{J F}$.

Inserting the conductance equation into Eq. (43) under saturated, linear groundwater flow conditions with the assumption of only small changes in the flow geometry ( $\lambda$ is constant) leads to

$\overline{S_{i}}=\lambda^{-1} \overline{J^{2}}=\lambda^{-1}\left[\operatorname{var}(J)+\bar{J}^{2}\right]$.

Thus, entropy production is related inversely to $\lambda$, linearly to $\operatorname{var}(J)$, and by a power of 2 to $\bar{J}$. If an estimate of $\lambda$ is available, $\overline{S_{i}}$ can be calculated from observations of $J$. In the more realistic case of variably saturated groundwater flow and/or varying flow geometry, Eq. (44) changes to

$\overline{S_{i}}=\overline{\lambda^{-1} J^{2}}=\operatorname{cov}\left(\lambda^{-1}, J^{2}\right)+\overline{\lambda^{-1}} \overline{J^{2}}$,

illustrating the same dependence of $\overline{S_{i}}$ on $\bar{\lambda}$ and $\bar{J}$ as before. The unknown covariance $\operatorname{cov}\left(\lambda^{-1}, J^{2}\right)$ may potentially be estimated from numerical experiments. 


\section{Summary and conclusions}

In this study, the method of inference based on the entropy balance equation was introduced. The theoretical basis is the explicit calculation of the internal microscale entropy production, which is used in the balance equation to solve for macroscale potentials, and thermodynamic forces and fluxes. The proposed method was illustrated with simple hydrologic cross sections of steady-state, variably saturated groundwater flow and a periodic source/sink (infiltration/evapotranspiration) at dynamic equilibrium.

The entropy balance equation is remarkable, because the equation unifies the macro- and microscale in one equation allowing the simultaneous application of two different movement equations, which are the conductance equation at the macroscale and Darcy's law/Richards' equation at the microscale, in this study. The derivations lead to expressions for macroscale variables that are a function of the entropy production (i.e., the internal fluctuations of the microscale flux-force relationships) and provide a thermodynamically consistent link between the two different scales. Therefore, the derivation provides a different theoretical perspective of variably saturated groundwater flow and new approaches for obtaining effective macroscale variables. The discussion suggests that these may be derived consistently for a hierarchy of scales. With the advent of high-performance computing in hydrology, there is strong potential for additional insight from hyper-resolution numerical experiments to explicitly calculate the internal entropy production. For example, existing and new averaging and upscaling laws may be tested and derived using series of numerical experiments with, e.g., varying subsurface heterogeneity configurations and boundary conditions. These experiments may also be useful in deriving new movement equations at the macroscale replacing empirical, calibrated parameterizations, and regionalization approaches.

The study is a contribution to the field of theoretical hydrology, providing a thermodynamic perspective of inference in hydrology. While inference of macroscale variables' necessitates explicit calculation of the entropy production and thus considerable computational resources, these resources are well invested: obtaining previously unknown macroscale parameters is at the center of the ubiquitous challenge of upscaling, and applying the proposed framework may help in finding general upscaling relationships over a hierarchy of scales. The connection to real-world observations needs to be established in the future, also with the help of numerical simulations. In the provided theoretical setting, the usefulness of the method for predictions is evident from the simple examples provide here, however, for real-world predictions this remains to be demonstrated.

Acknowledgements. I would like to thank the associate editor, Murugesu Sivapalan, and the reviewers Stan Schymanski, Martijn Westhoff, Erwin Zehe, one anonymous reviewer, and Claudius
Bürger for their constructive comments and suggestions during the review process. I also would like to thank Elbert Branscomb for the initial discussions and Axel Kleidon for more extensive discussions.

The article processing charges for this open-access

publication were covered by a Research

Centre of the Helmholtz Association.

Edited by: M. Sivapalan

\section{References}

Abe, S. and Okuyama, S.: Similarity between quantum mechanics and thermodynamics: Entropy, temperature, and Carnot cycle, Phys. Rev., 83, 021121, 2011.

del Jesus, M., Foti, R., Rinaldo, A., and Rodriguez-Iturbe, I.: Maximum entropy production, carbon assimilation, and the spatial organization of vegetation in river basins, P. Natl. Acad. Sci. USA, 109, 20837-20841, 2012.

Dewar, R.: Information theory explanation of the fluctuation theorem, maximum entropy production and self-organized criticality in non-equilibrium stationary states, J. Phys., 36, 631-641, 2003.

Dewar, R. C.: Maximum Entropy Production as an Inference Algorithm that Translates Physical Assumptions into Macroscopic Predictions: Don't Shoot the Messenger, Entropy, 11, 931-944, 2009.

Dewar, R. C.: Maximum entropy production and plant optimization theories, Phil. Trans. Roy. Soc., 365, 1429-1435, 2010.

Ehret, U., Gupta, H. V., Sivapalan, M., Weijs, S. V., Schymanski, S. J., Blöschl, G., Gelfan, A. N., Harman, C., Kleidon, A., Bogaard, T. A., Wang, D., Wagener, T., Scherer, U., Zehe, E., Bierkens, M. F. P., Di Baldassarre, G., Parajka, J., van Beek, L. P. H., van Griensven, A., Westhoff, M. C., and Winsemius, H. C.: Advancing catchment hydrology to deal with predictions under change, Hydrol. Earth Syst. Sci., 18, 649-671, doi:10.5194/hess-18-6492014, 2014.

Klausmeier, C. A.: Regular and irregular patterns in semiarid vegetation, Science, 284, 1826-1828, 1999.

Kleidon, A. and Schymanski, S.: Thermodynamics and optimality of the water budget on land: A review, Geophys. Res. Lett., 35, L20404, doi:10.1029/2008GL035393, 2008.

Kleidon, A., Zehe, E., Ehret, U., and Scherer, U.: Thermodynamics, maximum power, and the dynamics of preferential river flow structures at the continental scale, Hydrol. Earth Syst. Sci., 17, 225-251, doi:10.5194/hess-17-225-2013, 2013.

Kondepudi, D. and Prigogine, I.: Modern Thermodynamics: From Heat Engines to Dissipative Structures, 2nd Edition. Modern Thermodynamics: From Heat Engines to Dissipative Structures, 2nd Edition, Wiley, 552 pp., 2014.

Koutsoyiannis, D.: Entropy: From Thermodynamics to Hydrology, Entropy, 16, 1287-1314, 2014.

Kresse, G. and Hafner, J.: Ab-Initio Molecular-Dynamics Simulation of the Liquid-Metal Amorphous-Semiconductor Transition in Germanium, Phys. Rev., 49, 14251-14269, 1994.

Miedziejko, E. M. and Kedziora, A.: Impact of plant canopy structure on the transport of ecosystem entropy, Ecol. Model., 289, $15-25,2014$. 
Paillard, D. and Herbert, C.: Maximum Entropy Production and Time Varying Problems: The Seasonal Cycle in a Conceptual Climate Model, Entropy, 15, 2846-2860, 2013.

Porada, P., Kleidon, A., and Schymanski, S. J.: Entropy production of soil hydrological processes and its maximisation, Earth Syst. Dynam., 2, 179-190, doi:10.5194/esd-2-179-2011, 2011.

Schymanski, S. J., Kleidon, A., Stieglitz, M., and Narula, J.: Maximum entropy production allows a simple representation of heterogeneity in semiarid ecosystems, Phil. Trans. Roy. Soc., 365, 1449-1455, 2010.

Westhoff, M. C. and Zehe, E.: Maximum entropy production: can it be used to constrain conceptual hydrological models?, Hydrol. Earth Syst. Sci., 17, 3141-3157, doi:10.5194/hess-17-31412013, 2013.
Westhoff, M. C., Zehe, E., and Schymanski, S. J.: Importance of temporal variability for hydrological predictions based on themaximum entropy production principle, Geophys. Res. Lett., 41, 67-73, 2014.

Zehe, E., Ehret, U., Blume, T., Kleidon, A., Scherer, U., and Westhoff, M.: A thermodynamic approach to link self-organization, preferential flow and rainfall-runoff behaviour, Hydrol. Earth Syst. Sci., 17, 4297-4322, doi:10.5194/hess-17-4297-2013, 2013. 